University of South Carolina

Scholar Commons

2000

\title{
A Semianalytical Method for Predicting Primary and Secondary Current Density Distributions: Linear and Nonlinear Boundary Conditions
}

\author{
Dhanwa Thirumalai \\ Ralph E. White \\ University of South Carolina - Columbia, white@cec.sc.edu
}

Follow this and additional works at: https://scholarcommons.sc.edu/eche_facpub

Part of the Chemical Engineering Commons

\begin{abstract}
Publication Info
Published in Journal of the Electrochemical Society, Volume 147, Issue 5, 2000, pages 1636-1644.

(c) The Electrochemical Society, Inc. 2000. All rights reserved. Except as provided under U.S. copyright law, this work may not be reproduced, resold, distributed, or modified without the express permission of The Electrochemical Society (ECS). The archival version of this work was published in Subramanian, V.R. \& White, R.E. (2000). A Semi-analytical Method for Predicting Primary and Secondary Current Density Distributions: Linear and Non-linear Boundary Conditions. Journal of the Electrochemical Society, 147(5) 1636-1644.

Publisher's Version: http://dx.doi.org/10.1149/1.1393410
\end{abstract}

This Article is brought to you by the Chemical Engineering, Department of at Scholar Commons. It has been accepted for inclusion in Faculty Publications by an authorized administrator of Scholar Commons. For more information, please contact digres@mailbox.sc.edu. 


\title{
A Semianalytical Method for Predicting Primary and Secondary Current Density Distributions: Linear and Nonlinear Boundary Conditions
}

\author{
Venkat R. Subramanian* and Ralph E. White ${ }^{* *, z}$
}

Department of Chemical Engineering, University of South Carolina, Columbia, South Carolina 29208, USA

\begin{abstract}
A semianalytical solution technique is presented for solving Laplace's equation to obtain primary and secondary potential and current density distributions in electrochemical cells. The potential distribution inside a rectangle with the electrodes facing each other between two insulators is presented to illustrate the method. It is shown that the method yields analytic equations for the potential and the potential gradient along the lines. The unique attribute of the technique developed is that the solution once obtained is valid for nonlinear boundary conditions also. The procedure is applied to some realistic problems encountered in electrochemical engineering to illustrate the utility of the technique developed.
\end{abstract}

(C) 2000 The Electrochemical Society. S0013-4651(99)09-085-0. All rights reserved.

Manuscript submitted September 22, 1999; revised manuscript received December 20, 1999.

Potential distributions and their associated current density distributions (primary and secondary) are typically obtained by solving Laplace's equation. ${ }^{1-3}$ The methods used to solve Laplace's equation include analytic and numerical methods. Analytic methods (e.g., conformal mapping ${ }^{4}$ ) provide the maximum insight into the problem and usually yield closed-form potential and current density distributions. Unfortunately, analytic techniques are system specific and are often difficult to obtain. Numerical techniques are very general but usually give a numerical value for the potential at a particular location. We present here a semianalytical method (or analytic method of lines), which is for a two-spatial-coordinate problem analytic in $y$ and numerical in $x$; thereby the technique is more general than a particular analytic solution technique and gives better insight than numerical techniques for a certain class of problems (Laplace's equation, which has constant coefficients in at least one of the independent variables).

It is important to note that the method presented here for solving Laplace's equation in two spatial coordinates with nonlinear boundary conditions does not require iterations for interior node points as is usually the case. ${ }^{5}$ The reason for this is that our method does not require interior node points, but instead, only has node points in the boundaries. The nonlinearities of the boundary conditions are removed by solving for the constants that appear in the solution of Laplace's equation as explained in the following discussion.

The technique is an extension of the method presented by De Vidts and White, ${ }^{6}$ who presented the semianalytical method for solving the one-dimensional, unsteady-state diffusion equation. The semianalytical method presented by De Vidts and White consists of using the method of lines ${ }^{7}$ to solve the diffusion equation with finite differences used in the spatial direction. (This method was mentioned by Smith et al., ${ }^{8}$ but they did not present any results.) The resulting system of linear ordinary differential equations is then solved analytically using the matrix-exponential method. ${ }^{9}$ This technique is extended here to solve Laplace's equation with two spatial coordinates. The second derivative of the potential in the $x$ direction is cast into finite differences accurate to order $h^{2}(h=\Delta x)$, and the second derivative in the other direction is replaced by two first-order-derivative equations. The resulting system of ordinary differential equations is solved analytically using the matrix-exponential approach. The method requires determining constants of integration in a manner described previously by Subramanian et al. ${ }^{10}$ The method is illustrated by first solving Laplace's equation for a rectangle in which a cathode faces an anode between two insulators. The method employed for this simple case is then extended to other current-distribution problems.

\footnotetext{
* Electrochemical Society Student member

** Electrochemical Society Fellow.

${ }^{\mathrm{z}}$ E-mail: White@engr.sc.edu
}

\section{Semianalytical Method}

Consider the rectangle $\mathrm{ABCD}$ shown in Fig. 1 of dimensionless length $1(\mathrm{AB}=\mathrm{CD}=1)$ and dimensionless height $\mathrm{b}(\mathrm{AC}=\mathrm{BD}=$ $b$ ). The dimensionless governing equation for the dimensionless potential inside the rectangle is given by Laplace's equation

$$
\nabla^{2} \phi=\frac{\partial^{2} \phi}{\partial x^{2}}+\frac{\partial^{2} \phi}{\partial y^{2}}=0
$$

with the boundary conditions (at the insulators)

$$
\frac{\partial \phi}{\partial x}=0 \text { at } x=0 \text { and } x=1 \text { for } 0 \leq y \leq \mathrm{b}
$$

where $b$ is the height of the rectangle and

$$
\begin{gathered}
\phi=\phi_{\mathrm{a}}=0 \text { at } y=0 \text { for } 0 \leq x \leq 1 \text { (reversible anode) } \\
\phi=\phi_{\mathrm{c}}=10 \text { at } y=\mathrm{b} \text { for } 0 \leq x \leq 1 \text { (reversible cathode) }
\end{gathered}
$$

where $\phi_{\mathrm{a}}$ and $\phi_{\mathrm{c}}$ are the anode and cathode potentials. Note that anode is assigned a potential less than that of the cathode. The first

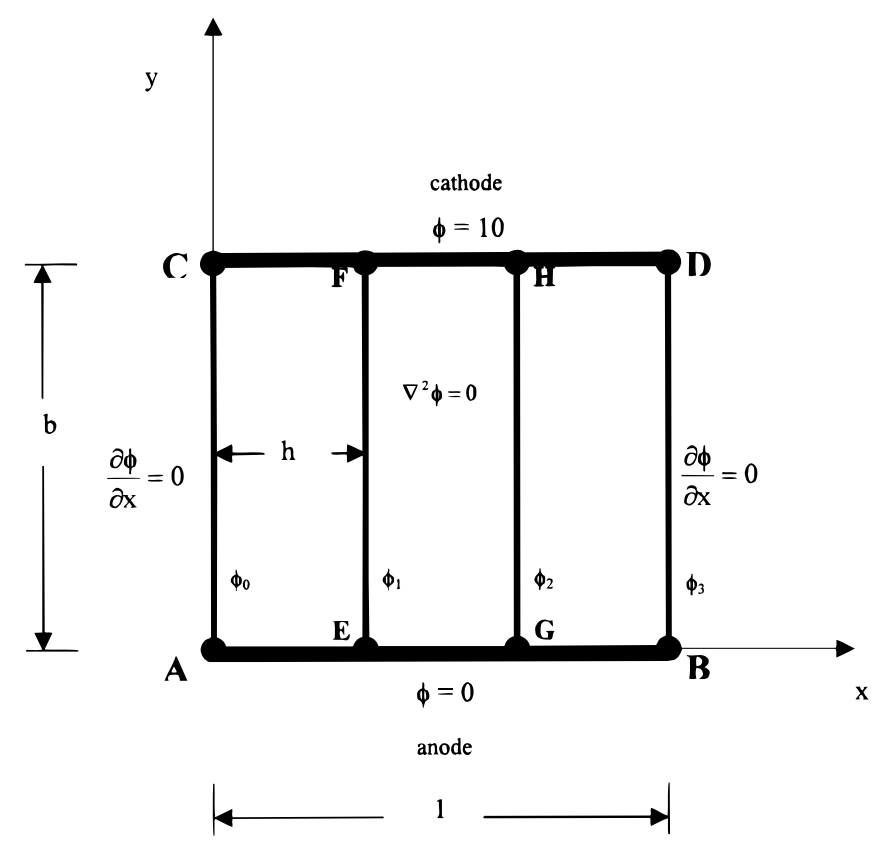

Figure 1. Dimensionless potential distribution inside a rectangle, node spacing in a semianalytical method. 
step in the semianalytical technique for this problem is to specify a grid as shown in Fig. 1, where two $(N=2)$ equally spaced interior node points ( $\mathrm{E}$ and $\mathrm{G}$ ) have been placed on the $x$ axis and two ( $\mathrm{F}$ and $\mathrm{H})$ on the $y=\mathrm{b}$ line. The step size in the $x$ direction is

$$
h=\Delta x=\frac{1}{N+1}
$$

which for this case $(N=2)$ gives $h=1 / 3$. As shown in Fig. 1 , there are four node points on the $x$ axis, and the rectangle is divided by four lines (AC, $\mathrm{EF}, \mathrm{GH}$, and $\mathrm{BD})$. The potentials of these four lines $(\mathrm{AC}, \mathrm{EF}, \mathrm{GH}$, and $\mathrm{BD})$ depend on $y$ and are represented as $\phi_{\mathrm{i}}(y), \mathrm{i}=$ $0,1, \ldots, N+1$. The boundary conditions (Eq. 2) are written in finitedifference form (accurate to the order $h^{2}$ )

$$
\frac{-\phi_{2}+4 \phi_{1}-3 \phi_{0}}{2 h}=0 \text { for } 0 \leq y \leq \mathrm{b} \text { at } x=0
$$

and

$$
\frac{\phi_{1}-4 \phi_{2}+3 \phi_{3}}{2 h}=0 \text { for } 0 \leq y \leq \mathrm{b} \text { at } x=1
$$

The next step is to cast in finite-difference form the second derivative of $\phi$ with respect to $x$ in Laplace's equation and to retain the second derivative in $y$ for $\phi_{1}$ and $\phi_{2}$

$$
\frac{d^{2} \phi_{1}}{d y^{2}}+\frac{\phi_{0}-2 \phi_{1}+\phi_{2}}{h^{2}}=0
$$

and

$$
\frac{d^{2} \phi_{2}}{d y^{2}}+\frac{\phi_{1}-2 \phi_{2}+\phi_{3}}{h^{2}}=0
$$

By using Eq. 6 and 7, Eq. 8 and 9 become

$$
\frac{d^{2} \phi_{1}}{d y^{2}}=\frac{2}{3} \frac{\phi_{1}-\phi_{2}}{h^{2}}
$$

Thus for $N=2$, we have 4 (i.e., $2 N)$ dependent variables $\left(Y_{1}, Y_{2}, Y_{3}\right.$, and $Y_{4}$ ). Note that these dependent variables consist of the potential and its derivatives in $y$ along the lines EF and GH. Equations 13-16 can be written in matrix form

$$
\frac{d \mathbf{Y}}{d y}=\mathbf{A Y}
$$

where

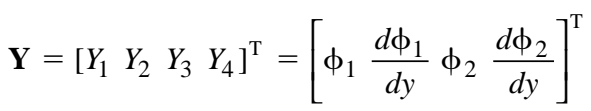

The coefficient matrix is given by

$$
\mathbf{A}=\left[\begin{array}{cccc}
0 & 1 & 0 & 0 \\
\frac{2}{3 h^{2}} & 0 & -\frac{2}{3 h^{2}} & 0 \\
0 & 0 & 0 & 1 \\
-\frac{2}{3 h^{2}} & 0 & \frac{2}{3 h^{2}} & 0
\end{array}\right]
$$

Equation 17 can be solved in two steps as presented earlier by Subramanian et al. ${ }^{10}$ The solution for the vector differential Eq. 17 can be written as ${ }^{9}$

$$
\mathbf{Y}=\exp (\mathbf{A} y) \mathbf{Y}_{0}
$$

where

$$
\mathbf{Y}_{0}=\left[\begin{array}{llll}
Y_{1} & Y_{2} & Y_{3} & Y_{4}
\end{array}\right]_{y=0}^{\mathrm{T}}=\left[\phi_{1} \frac{d \phi_{1}}{d y} \phi_{2} \frac{d \phi_{2}}{d y}\right]_{y=0}^{\mathrm{T}}
$$

We call Eq. 20 the semianalytical solution because we discretized in $x$ and integrated analytically in $y$. The exponential matrix in Eq. 20 can be calculated using Maple $\mathrm{V}^{\circledR}$. For $N=2$ node points, the exponential matrix as calculated from Maple is

$$
\exp (\mathbf{A} y)=\left[\begin{array}{cccc}
0.5+0.5 \operatorname{ch}(3.46 y) & 0.5 y+0.144 \operatorname{sh}(3.46 y) & 0.5-0.5 \operatorname{ch}(3.46 y) & 0.5 y-0.144 \operatorname{sh}(3.46 y) \\
1.73 \operatorname{sh}(3.46 y) & 0.5+0.5 \operatorname{ch}(3.46 y) & -1.73 \operatorname{sh}(3.46 y) & 0.5-0.5 \operatorname{ch}(3.46 y) \\
0.5-0.5 \operatorname{ch}(3.46 y) & 0.5 y-0.144 \operatorname{sh}(3.46 y) & 0.5+0.5 \operatorname{ch}(3.46 y) & 0.5 y+0.144 \operatorname{sh}(3.46 y) \\
-1.73 \operatorname{sh}(3.46 y) & 0.5-0.5 \operatorname{ch}(3.46 y) & 1.73 \operatorname{sh}(3.46 y) & 0.5+0.5 \operatorname{ch}(3.46 y)
\end{array}\right]
$$

and

$$
\frac{d^{2} \phi_{2}}{d y^{2}}=-\frac{2}{3} \frac{\phi_{1}-\phi_{2}}{h^{2}}
$$

The next step is to change the second-order equations, Eq. 10 and 11, into a system of first-order equations. To do this, let

$$
Y_{1}=\phi_{1} ; Y_{2}=\frac{d \phi_{1}}{d y} ; Y_{3}=\phi_{2} ; Y_{4}=\frac{d \phi_{2}}{d y}
$$

The first-order system of equations that results is

$$
\begin{gathered}
\frac{d Y_{1}}{d y}=Y_{2} \quad \text { (by definition) } \\
\frac{d Y_{2}}{d y}=\frac{2}{3 h^{2}} Y_{1}-\frac{2}{3 h^{2}} Y_{3} \quad \text { (from Eq. 10) } \\
\frac{d Y_{3}}{d y}=Y_{4} \quad \text { (by definition) } \\
\frac{d Y_{4}}{d y}=-\frac{2}{3 h^{2}} Y_{1}+\frac{2}{3 h^{2}} Y_{3} \quad \text { (from Eq. 11) }
\end{gathered}
$$

Note that in Eq. $22 \mathrm{sh}$ and ch are used for hyperbolic sines and cosines, respectively, for brevity. It should be noted that a closedform solution is obtained (Eq. 20) without using the boundary condition in $y$ (Eq. 3 and 4) and hence, the solution obtained is valid for any boundary condition at $y=0$ and $y=1$.

For solving the particular boundary-value problem (BVP) for the boundary conditions given by Eq. 3 and 4, the first step is using the boundary values for $\phi_{1}$ and $\phi_{2}$ at $y=0$ with the derivative of $\phi_{1}$ and $\phi_{2}\left(d \phi_{1} / d y\right.$ and $\left.d \phi_{2} / d y\right)$ at $y=0$ treated as constants $c_{1}$ and $c_{2}$, respectively. The second step is to apply the boundary condition for $\phi_{1}$ and $\phi_{2}$ at $y=\mathrm{b}$ to solve for the constants $c_{1}$ and $c_{2}$, which are then substituted back into the analytic solution (Eq. 20). The boundary conditions on $\phi_{1}$ and $\phi_{2}$ at $y=0$ are

$$
\phi_{1}=\phi_{2}=0 \text { at } y=0
$$

i.e.

$$
Y_{1}=Y_{3}=0 \text { at } y=0
$$

Let the derivatives of $\phi$ in $y$ at $y=0$ be

$$
\frac{d \phi_{1}}{d y}(0)=Y_{2}(0)=c_{1}
$$


and

$$
\frac{d \phi_{2}}{d y}(0)=Y_{4}(0)=c_{2}
$$

Hence, the initial condition vector $\left(\mathbf{Y}_{0}\right)$ becomes

$$
\begin{aligned}
\mathbf{Y}_{0}=\left[\begin{array}{llll}
Y_{1} & Y_{2} & Y_{3} & Y_{4}
\end{array}\right]_{y=0}^{\mathrm{T}}=\left[\begin{array}{ll}
\phi_{1} \frac{d \phi_{1}}{d y} & \phi_{2} \frac{d \phi_{2}}{d y}
\end{array}\right]_{y=0}^{\mathrm{T}} & \\
& =\left[\begin{array}{llll}
0 & c_{1} & 0 & c_{2}
\end{array}\right]^{\mathrm{T}}
\end{aligned}
$$

Substituting these values into Eq. 20 yields

$$
\mathbf{Y}=\left[\begin{array}{c}
{[0.5 y+0.144 \operatorname{sh}(3.46 y)] c_{1}+[0.5 y-0.144 \operatorname{sh}(3.46 y)] c_{2}} \\
{[0.5+0.5 \operatorname{ch}(3.46 y)] c_{1}+[0.5-0.5 \operatorname{ch}(3.46 y)] c_{2}} \\
{[0.5 y-0.144 \operatorname{sh}(3.46 y)] c_{1}+[0.5 y+0.144 \operatorname{sh}(3.46 y)] c_{2}} \\
{[0.5 y-0.5 \operatorname{ch}(3.46 y)] c_{1}+[0.5 y+0.5 \operatorname{ch}(3.46 y)] c_{2}}
\end{array}\right]
$$

Thus, a general solution is obtained with $c_{1}$ and $c_{2}$ as constants. This solution (Eq. 28) evaluated at $y=1$ is

$$
\mathbf{Y}_{y=1}=\left[\begin{array}{c}
\phi_{1} \\
\frac{\partial \phi_{1}}{\partial y} \\
\phi_{2} \\
\frac{\partial \phi_{2}}{\partial y}
\end{array}\right]_{y=1}=\left[\begin{array}{c}
2.8 c_{1}-1.8 c_{2} \\
8.45 c_{1}-7.45 c_{2} \\
-1.8 c_{1}+2.8 c_{2} \\
-7.45 c_{1}+8.45 c_{2}
\end{array}\right]
$$

The values of the constants can be obtained by using the boundary condition (Eq. 4) at $y=1$ (i.e., $\phi_{1}=\phi_{2}=\phi_{\mathrm{b}}$ )

$$
\begin{aligned}
\mathbf{Y}_{y=1}=\left[\begin{array}{llll}
Y_{1} & Y_{2} & Y_{3} & Y_{4}
\end{array}\right]_{y=1}^{\mathrm{T}}=\left[\phi_{1} \frac{d \phi_{1}}{d y} \phi_{2} \frac{d \phi_{2}}{d y}\right]_{y=1}^{\mathrm{T}} \\
=\left[\phi_{\mathrm{b}} \frac{d \phi_{1}}{d y} \phi_{\mathrm{b}} \frac{d \phi_{2}}{d y}\right]_{y=1}^{\mathrm{T}}
\end{aligned}
$$

Comparison of Eq. 29 and 30 show that

$$
2.8 c_{1}-1.8 c_{2}=\phi_{\mathrm{b}}
$$

and

$$
-1.8 c_{1}+2.8 c_{2}=\phi_{\mathrm{b}}
$$

Solving Eq. 31 and 32 we get

$$
c_{1}=c_{2}=\phi_{\mathrm{b}}=10
$$

Substituting these constants $\left(c_{1}\right.$ and $\left.c_{2}\right)$ into Eq. 28 yields the complete solution

$$
\begin{aligned}
& \mathbf{Y}=\left[\begin{array}{llll}
Y_{1} & Y_{2} & Y_{3} & Y_{4}
\end{array}\right]^{\mathrm{T}}=\left[\phi_{1} \frac{d \phi_{1}}{d y} \phi_{2} \frac{d \phi_{2}}{d y}\right]^{\mathrm{T}} \\
& =\left[\begin{array}{llll}
10 y & 10 & 10 y & 10
\end{array}\right]^{\mathrm{T}}
\end{aligned}
$$

The expected linear potential profile is obtained. Note that a solution is obtained simultaneously for both the potential and its derivative in $y$, which is an added benefit of this method. Once $\phi_{1}(y)$ and $\phi_{2}(y)$ are obtained after obtaining the boundary conditions, $\phi_{0}(y)$ and $\phi_{3}(y)$ can be found from Eq. 6 and 7, respectively. This example also shows that our technique is numerical in $x$ and analytic in $y$.

\section{Boundary Conditions}

The solution obtained (Eq. 28) is also valid for linear and nonlinear boundary conditions at the cathode. For example, if the boundary condition at the cathode is given by linear kinetics

$$
\frac{\partial \phi}{\partial y}=J_{\mathrm{c}}(10-\phi) \text { at } y=1 \text { for } 0 \leq x \leq 1 \text { (cathode) }
$$

where $J_{\mathrm{c}}$ is a dimensionless cathodic polarization parameter. In this case, Eq. 31 and 32 become

$$
8.45 c_{1}-7.45 c_{2}=J_{c}\left(10-2.8 c_{1}+1.8 c_{2}\right)
$$

and

$$
-7.45 c_{1}+8.45 c_{2}=J_{c}\left(10+1.8 c_{1}-2.8 c_{2}\right)
$$

Solving Eq. 36 and 37 for $c_{1}$ and $c_{2}$ yields

$$
c_{1}=c_{2}=\frac{J_{\mathrm{c}}}{1+J_{\mathrm{c}}} 10
$$

These constants ( $c_{1}$ and $c_{2}$ from Eq. 38) can be substituted into Eq. 28 to obtain the potential profiles for linear kinetics at the cathode. The solution obtained is also valid for nonlinear boundary conditions. For example, if the cathode kinetics are given by the nonlinear Butler-Volmer equation

$$
\frac{\partial \phi}{\partial y}=J_{\mathrm{c}}\{\exp [0.5(10-\phi)]-\exp [-0.5(10-\phi)]\}
$$

Equations 31 and 32 become

$$
\begin{aligned}
8.45 c_{1}-7.45 c_{2}=J_{\mathrm{c}}\left[\operatorname { e x p } \left(-1.4 c_{1}\right.\right. & \left.+0.9 c_{2}+5\right) \\
& \left.-\exp \left(-5+1.4 c_{1}-0.9 c_{2}\right)\right]
\end{aligned}
$$

and

$$
\begin{aligned}
-7.45 c_{1}+8.45 c_{2}=J_{\mathrm{c}}\left[\exp \left(0.9 c_{1}-1.4 c_{2}+5\right)\right. & \\
& \left.-\exp \left(-5-0.9 c_{1}+1.4 c_{2}\right)\right]
\end{aligned}
$$

These nonlinear algebraic equations (Eq. 40 and 41) can be solved easily using Maple. For example, if $J_{\mathrm{c}}=1$, using Maple's fsolve command, these equations yield

$$
c_{1}=c_{2}=6.27 \text { for } J_{\mathrm{c}}=1
$$

If $J_{\mathrm{c}}=10$, these two equations can be solved by using Maple to give

$$
c_{1}=c_{2}=9.12 \text { for } J_{\mathrm{c}}=10
$$

These values for $c_{1}$ and $c_{2}$ can be substituted into Eq. 28 to get the potential profiles for this case where the boundary condition at the cathode is nonlinear. As the polarization parameter $J_{\mathrm{c}}$ is increased, the primary current distribution (Eq. 33) is approached, as expected. For a given boundary condition at $x=0$ and $x=1$, the problem is solved generally and is valid for any boundary condition on the cathodes and anodes placed at $y=0$ and $y=1$. Note that our solution for a nonlinear boundary condition was obtained easily by merely solving for two $(N=2)$ nonlinear algebraic equations (Eq. 40 and 41). Similar procedure can be used for linear kinetics and ButlerVolmer kinetics expressions at the anode.

\section{Hull Cell}

Consider a Hull cell as shown in Fig. 2. The boundary conditions at the insulators and the anode $(y=0)$ are the same, while the boundary condition at the cathode is given by

$$
\phi=10 \text { at } y=1+0.5 x \text { (on the cathode) }
$$

Even for this BVP, the solution obtained (Eq. 28) is still valid. Substituting the numerical value for the position of the cathode we get 


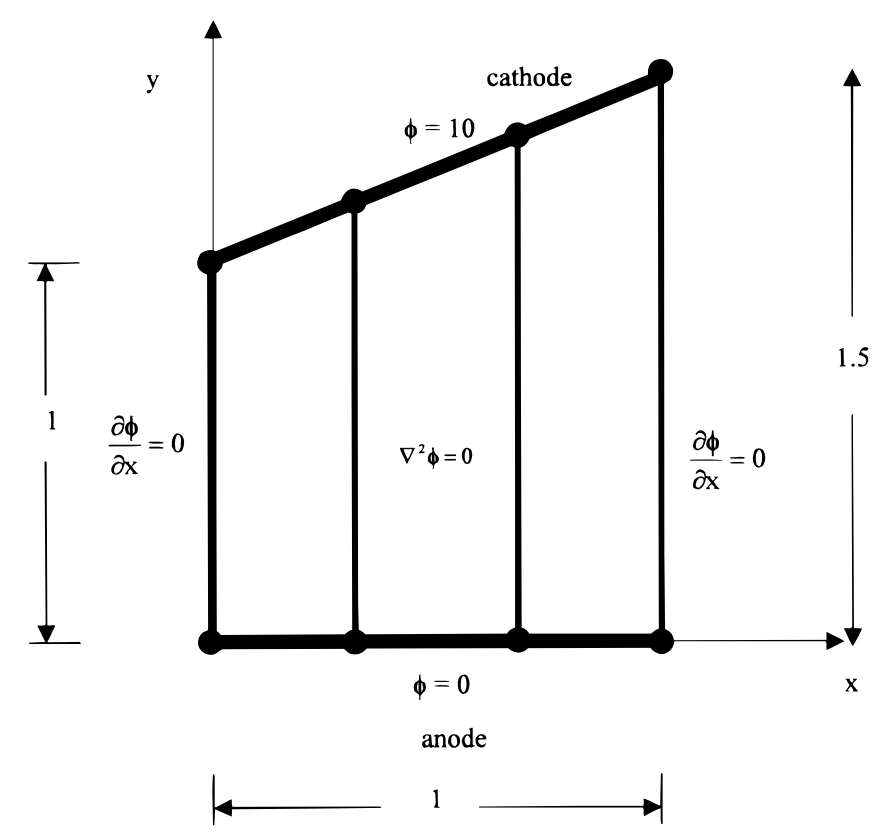

Figure 2. Semianalytical method for a Hull cell.

$$
\mathbf{Y}_{y=1+0.5 x}=\left[\begin{array}{c}
\phi_{1} \\
\frac{\partial \phi_{1}}{\partial y} \\
\phi_{2} \\
\frac{\partial \phi_{2}}{\partial y}
\end{array}\right]_{y=1+0.5 x}=\left[\begin{array}{c}
4.69 c_{1}-3.52 c_{2} \\
14.73 c_{1}-13.73 c_{2} \\
-6.64 c_{1}+7.98 c_{2} \\
-24.85 c_{1}+25.85 c_{2}
\end{array}\right]_{[45]}
$$

Using the boundary condition 44 with 45 , the constants are solved as before as

$$
c_{1}=8.21 \text { and } c_{2}=8.09
$$

Substituting these constants, potential profiles are obtained. The current distribution at the anode (for unit conductivity $k$ ) is plotted in Fig. 3 using $N=10$ node points. The time taken for finding the exponential matrix for this problem was $10 \mathrm{~s}$ using a Pentium 333 $\mathrm{MHz}$ processor. The time taken for solving the ten linear equations for the constants was around $2 \mathrm{~s}$. Hence, the total time taken was around $13 \mathrm{~s}$ using Maple to produce Fig. 3.

Suppose the boundary condition at the anode of the Hull cell is given by the linear kinetics expression

$$
\frac{\partial \phi}{\partial y}=J_{\mathrm{a}} \phi \quad \text { at } y=0
$$

Now the Laplace equation has to be solved with the insulator boundary conditions at $x=0$ and $x=1$ and Eq. 44 and 47 . Even now, the BVP need not be solved once again. The exponential matrix given by Eq. 22 is still valid. The initial condition at $y=0$ is taken as (based on Eq. 47)

$$
\begin{aligned}
\mathbf{Y}_{0}=\left[\begin{array}{llll}
Y_{1} & Y_{2} & Y_{3} & Y_{4}
\end{array}\right]_{y=0}^{\mathrm{T}}=\left[\begin{array}{ll}
\phi_{1} \frac{d \phi_{1}}{d y} & \left.\phi_{2} \frac{d \phi_{2}}{d y}\right]_{y=0}^{\mathrm{T}} \\
& =\left[\begin{array}{llll}
p_{1} & J_{\mathrm{a}} p_{1} & p_{2} & J_{\mathrm{a}} p_{2}
\end{array}\right]^{\mathrm{T}}
\end{array}\right.
\end{aligned}
$$

where $p_{1}$ and $p_{2}$ are the unknown potentials at the two node points. Substituting this initial-value vector into Eq. 20, an analytic solution similar to Eq. 28 is obtained. When the solution is evaluated at the cathode

$$
\begin{aligned}
\mathbf{Y}_{y=1+0.5 x}=\left[\begin{array}{c}
\phi_{1} \\
\frac{\partial \phi_{1}}{\partial y} \\
\phi_{2} \\
\frac{\partial \phi_{2}}{\partial y}
\end{array}\right]_{y=1+0.5 x} \\
=\left[\begin{array}{c}
14.73 p_{1}+4.69 J_{\mathrm{a}} p_{1}-13.73 p_{2}-3.52 J_{\mathrm{a}} p_{2} \\
49.27 p_{1}+14.73 J_{\mathrm{a}} p_{1}-49.27 p_{2}-13.73 J_{\mathrm{a}} p_{2} \\
-24.85 p_{1}-6.64 J_{\mathrm{a}} p_{1}+25.85 p_{2}+7.98 J_{\mathrm{a}} p_{2} \\
-87.78 p_{1}-24.85 J_{\mathrm{a}} p_{1}+87.78 p_{2}+25.85 J_{\mathrm{a}} p_{2}
\end{array}\right]
\end{aligned}
$$

This equation can be solved with the boundary condition (for a particular value of $J_{\mathrm{a}}$ ) to solve the constants $p_{1}$ and $p_{2}$. Using the exponential matrix developed for Fig. 3, by merely recalculating the constants, the linear-kinetics secondary current distribution at the anode is obtained and plotted in Fig. 4 (for unit conductivity) for various values of the polarization parameter $J_{\mathrm{a}}$. Note that as $J_{\mathrm{a}}$ increases, primary current density distribution is approached (Fig. 3).

Let the boundary condition at the anode be given by the nonlinear Butler-Volmer boundary condition

$$
-\frac{\partial \phi}{\partial y}=J_{\mathrm{a}}[\exp (-0.5 \phi)-\exp (0.5 \phi)] \text { at } y=0
$$

Again, there is no need to solve the BVP once again; using the exponential matrix obtained for Fig. 3, by merely recalculating the constants the secondary nonlinear current distribution in the anode is obtained and plotted in Fig. 5. Maple is used to solve the nonlinear algebraic equations arising out of the nonlinear boundary condition. The Newton-Raphson technique can be used to solve the system of

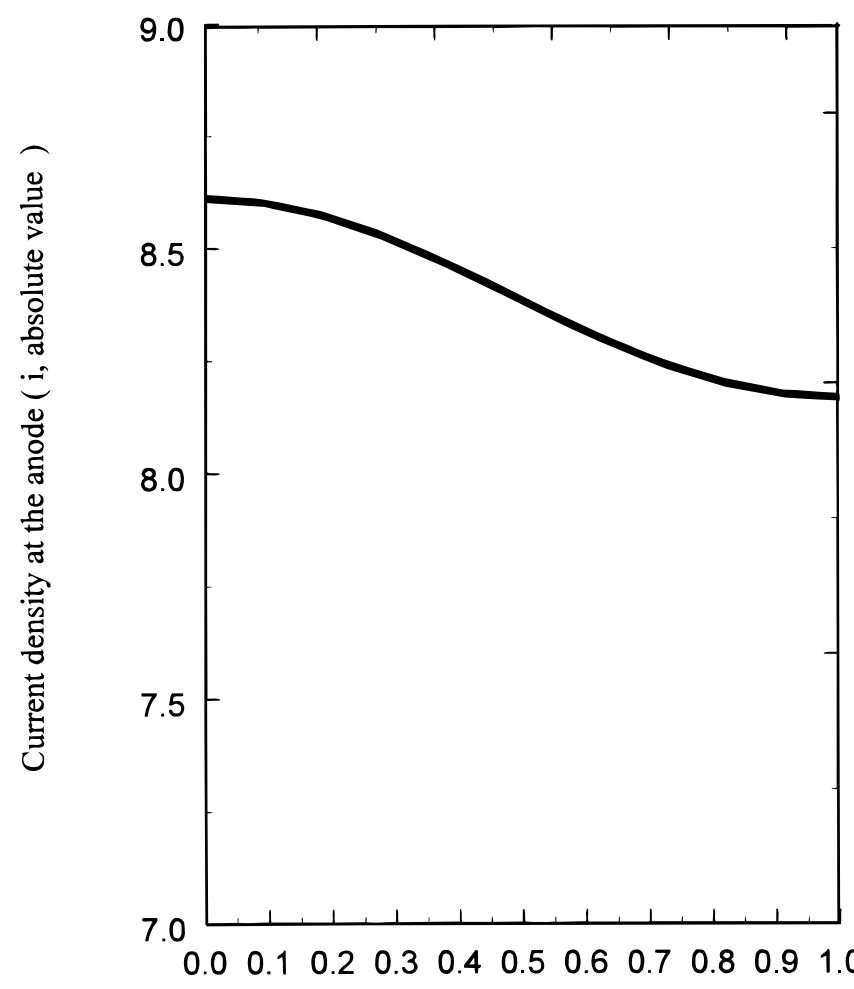

Dimensionless distance $\mathrm{x}$

Figure 3. Primary current density distribution in a Hull cell. 
nonlinear equations more efficiently (not reported here, but can be obtained upon request from the authors). Time taken for solving for a particular value of $J_{\mathrm{a}}=1$ is around $4 \mathrm{~s}$. Similarly, the profiles for different values of parameters are obtained. As seen in Fig. 5, for higher values of the polarization parameter $J_{\mathrm{a}}$, the primary current distribution (Fig. 3) is approached, as expected. Note that by using the same exponential matrix for Fig. 3 (time taken $=20 \mathrm{~s}$ ), by solving for constants only (each curve in Fig. 5 takes only 4 s to solve for the constants), the secondary nonlinear current distribution on the anode is obtained. The utility of the technique developed is demonstrated further in later sections. The semianalytical technique developed can be generalized for any number of node points, as illustrated in Ref. 11.

\section{Current Density Distribution in a Curvilinear Hull Cell}

Consider current flow between planar electrodes placed on two radii of an annular section, ${ }^{12}$ forming a cell with concentric cylindrical walls (Fig. 6). The curved walls are insulators. The anode is reversible, and the cathodic reaction kinetics expression is linear. The governing equation in dimensionless form is

$$
\frac{1}{\rho} \frac{\partial}{\partial \rho}\left(\rho \frac{\partial \psi}{\partial \rho}\right)+\frac{1}{\rho^{2}} \frac{\partial^{2} \psi}{\partial \theta^{2}}=0
$$

with the boundary conditions

$$
\begin{gathered}
\frac{\partial \psi}{\partial \rho}=0 \quad \text { at } \rho=1 \text { and } \rho=3 \text { (insulator) } \\
\psi=0 \quad \text { at } \theta=\frac{\pi}{2} \text { (reversible anode) }
\end{gathered}
$$

and

$$
\psi-\frac{P}{\rho} \frac{\partial \psi}{\partial \theta}=1 \quad \text { at } \theta=0 \text { (cathode with linear kinetics) [54] }
$$

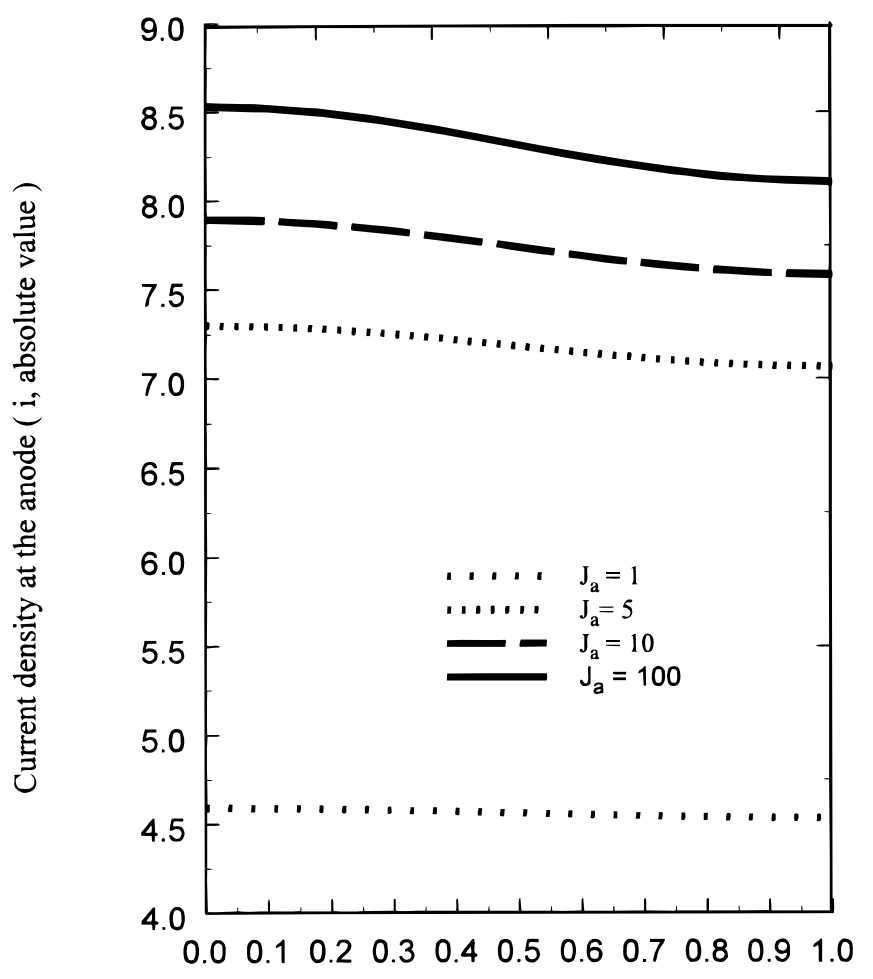

\section{$\mathbf{x}$, dimensionless distance}

Figure 4. Linear kinetics-secondary current density distribution in a Hull cell, effect of polarization parameter $J_{\mathrm{a}}$.

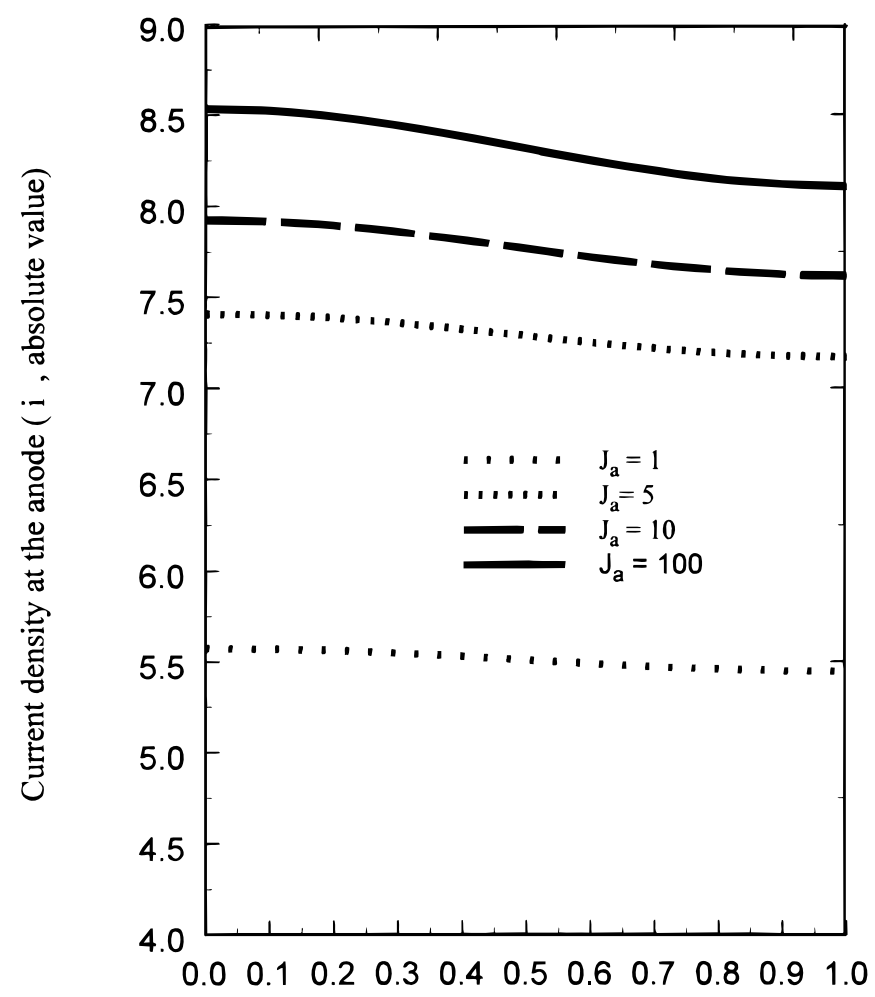

$\mathbf{x}$, dimensionless distance

Figure 5. Nonlinear secondary current density distribution in a Hull cell, semianalytical method for Butler-Volmer boundary conditions.

This BVP is chosen to illustrate the validity of our technique for a different coordinate system other than Cartesian. This BVP can be solved using our semianalytical technique by discretizing Eq. 51 in $\rho$ and integrating analytically in $\theta$. Once the solution $\psi(\rho, \theta)$ is obtained, the current density distribution along the cathode can be obtained as

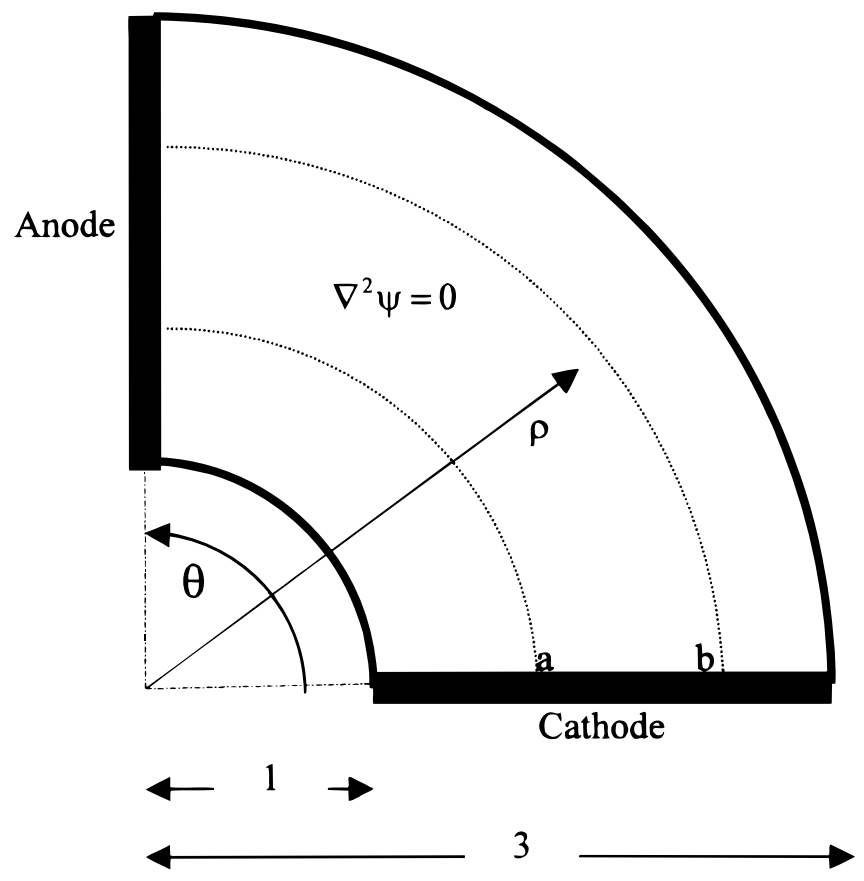

Figure 6. Semianalytical method for a curvilinear Hull cell. 


$$
i=\frac{1}{\rho} \frac{\partial \psi}{\partial \theta}_{\theta=0}
$$

The normalized current density along the cathode using $N=6$ is shown in Fig. 7 with $P$ as a parameter, which agrees with the figure presented by Lee and Chapman. ${ }^{12}$ The time taken with Maple to solve this BVP is around $20 \mathrm{~s}$ (for the entire program). All the plots given in Ref. 12 for different values of the polarization parameter $P$ were completely reproduced with our solution technique by merely solving the BVP only once with $P$ as a parameter in the boundary condition. Again it is worth repeating that our method yields values for $\psi$ and $i$ which are functions of the parameter $P$. This is particularly useful for parameter estimation (or optimization) studies because the computation time required to determine $P$ given the current density would be significantly less compared to a numerical technique, which would require a completely new solution for each value of $P$. Finally, the solution obtained can be modified easily for a change in dimension (radius, height, etc.).

\section{Thin-Layer Galvanic Cells}

Consider the thin-layer galvanic cell shown in Fig. $8,{ }^{13}$ the Laplace equation can be written in dimensionless form as ${ }^{13}$

$$
\frac{\partial^{2} \phi}{\partial y^{2}}+\epsilon^{2} \frac{\partial^{2} \phi}{\partial x^{2}}=0
$$

where $\epsilon=W / L$ is the aspect ratio, with the insulator boundary conditions at $x=0,1$ and $y=1$. The boundary condition at $y=0$ is given by

$$
\frac{\partial \phi}{\partial y}=J_{\mathrm{a}} \epsilon^{2} \phi 0 \leq x \leq a \text { at the anode }
$$

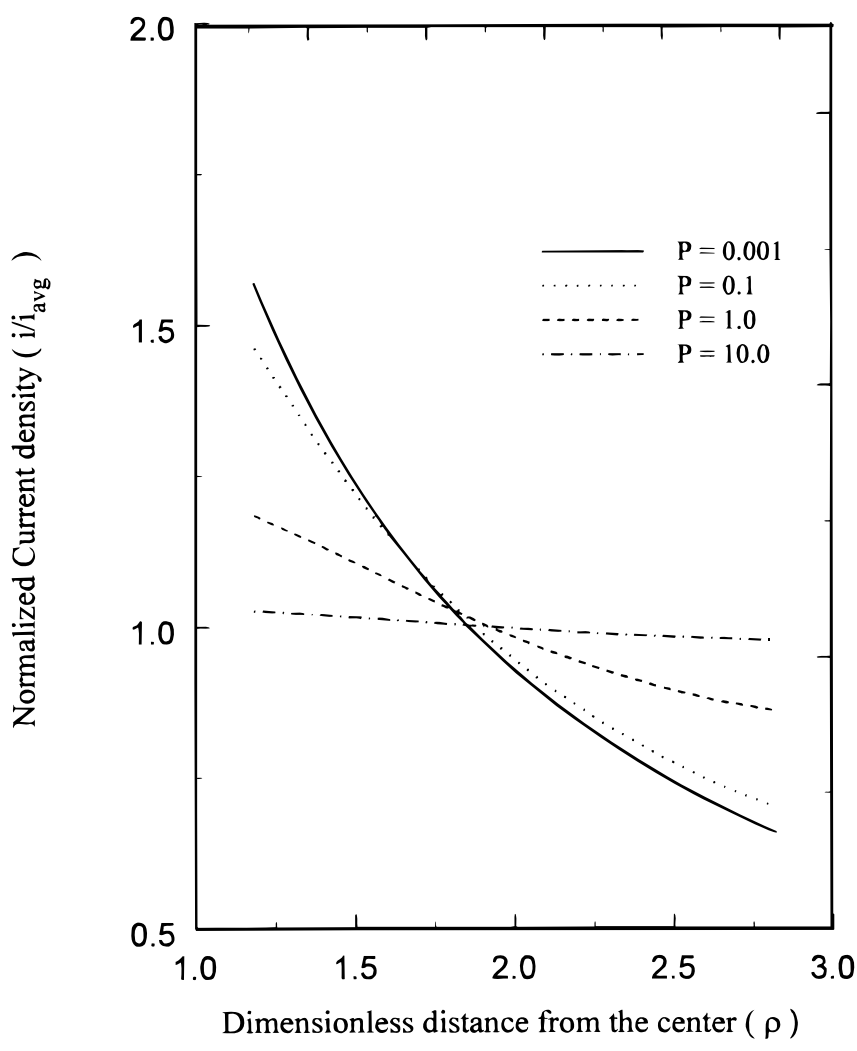

Figure 7. Secondary current distribution on a curvilinear Hull cell for different values of the polarization parameter $P$.

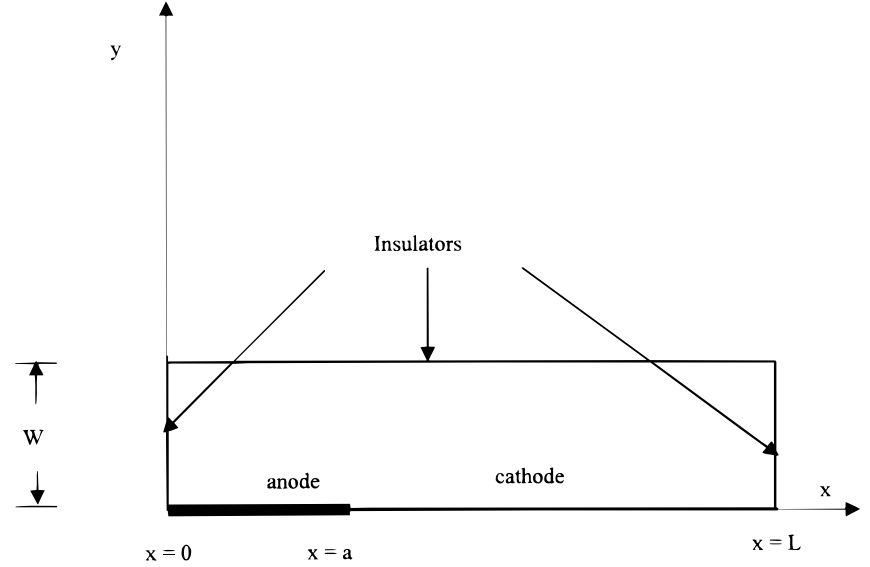

Figure 8. Thin-layer galvanic cell, semianalytical method for multiple regions.

and

$$
\frac{\partial \phi}{\partial y}=J_{\mathrm{c}} \epsilon^{2}(\phi-1) a \leq x \leq 1 \text { at the cathode }
$$

where $a$ is the ratio of anode length to the total length of anode and cathode. This example is chosen to illustrate the validity of our technique for a domain containing more than one region (of equal height). Now $N$ interior node points are chosen in the anode and $M$ interior node points are chosen in the cathode. The step sizes in the anode and cathode domain are given by

$$
h_{\text {anode }}=\frac{a}{N+1} \text { and } h_{\text {cathode }}=\frac{1-a}{M+1}
$$

Potentials at $x=0$ and $x=1$ are eliminated using the boundary conditions as before. Now the potential at the surface $(x=a)$ is expressed in terms of the potentials at the other node points using the continuity of flux as

$$
\begin{aligned}
& \frac{\partial \phi}{\partial x_{\text {anode },-}}=\frac{\partial \phi}{\partial x_{\text {cathode },+}} \\
& \text { or } \\
& \frac{1}{2} \frac{\phi_{N+1}-4 \phi_{N}+3 \phi_{N-1}}{h_{\text {anode }}}=\frac{1}{2} \frac{-\phi_{N+3}+4 \phi_{N+2}-3 \phi_{N+1}}{h_{\text {cathode }}}
\end{aligned}
$$

Only potentials at the $N$ interior node points in the anode and $M$ in the cathode are solved. The procedure is the same as before. Results for $N=M=4$ for $a=0.2$ and $\epsilon=10^{-3}$ are plotted in Fig. 9 and agree well with the results reported in the literature. Secondary current density distributions for different values of the polarization parameters are plotted in Fig. 9. Again, once the matrix exponential is found for the governing equation (Eq. 56), the secondary current distribution for nonlinear Butler-Volmer kinetics can be found. The total Maple time taken was around $80 \mathrm{~s}$. Also a similar BVP arising in a cylindrical thin-layer galvanic cell ${ }^{13}$ can be solved easily using our technique.

\section{Infinite Domains}

The technique developed in the previous sections can be extended to semi-infinite and infinite domains. Consider two plane electrodes of length $L$, each separated by a distance $H$ apart, placed opposite in the walls of an insulating flow channel. ${ }^{1}$ An analytic solution was presented by Newman ${ }^{1}$ for this geometry. Near the edge of the electrode the primary current density is infinite. This problem was solved numerically by Rousar et al. ${ }^{14}$ They changed the infinite domain into a finite domain by using a variable transformation

$$
X=1-\exp \left(-\frac{2 x}{L} \ln 2\right)
$$




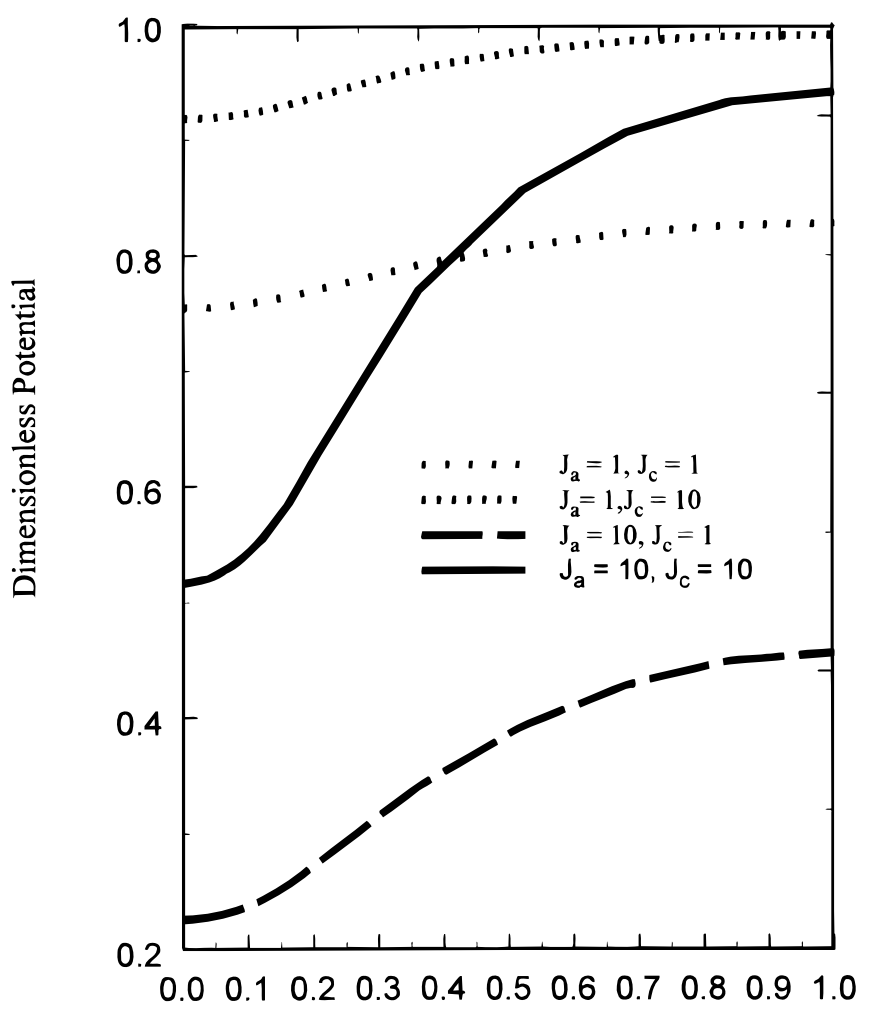

Dimensionless distance, $\mathrm{X}$

Figure 9. Linear secondary current density distribution in a thin-layer galvanic cell, effect of polarization parameter $J_{\mathrm{c}}$.

When this transformation is substituted into the Laplace equation (Eq. 1), only derivatives with respect to $x$ change. Therefore, the potentials can be integrated using the exponential matrix in $y$ direction using our technique. They also exploited the symmetry conditions and used a successive overrelaxation procedure to solve numerically the transformed problem. They compared their numerical results with the exact analytic solution in Fig. 6.2.8 of their book. They used 1587 node points to obtain their results. We solved the same BVP using our technique with 25 node points in the $x$ axis (20 on the anode and only 5 on the insulator, see the previous section for choice of node points). Our results (Fig. 10) are in excellent agreement with the analytic solution given in Ref. 1 . We were able to obtain the current density distribution (normalized to the numerical average) as a function of the aspect ratio $2 H / L$. The flexibility of our method regarding the dimensions helped us to obtain all the plots in Fig. 10 by solving the BVP just once and merely recalculating the constants (as explained previously). The time taken by Maple for running this BVP was around 5 min.

\section{Disk Electrode}

The primary current density distribution on a rotating-disk electrode (RDE) can be found using our technique. The potential is governed by Laplace's equation (in cylindrical coordinates). This BVP was elegantly solved by Newman ${ }^{4}$ using elliptic coordinates. However, for our technique, the transformation to elliptic coordinates is not useful, as illustrated in the next section. The boundary conditions are given by

$$
\begin{aligned}
& \phi=1 \quad \text { at } z=0, r \leq 1 \\
& \frac{\partial \phi}{\partial z}=0 \text { at } z=0, r>1 \text { and } \frac{\partial \phi}{\partial r}=0 \text { at } r=0 \\
& \phi \rightarrow 0 \text { as } r \rightarrow \infty \text { or } z \rightarrow \infty
\end{aligned}
$$

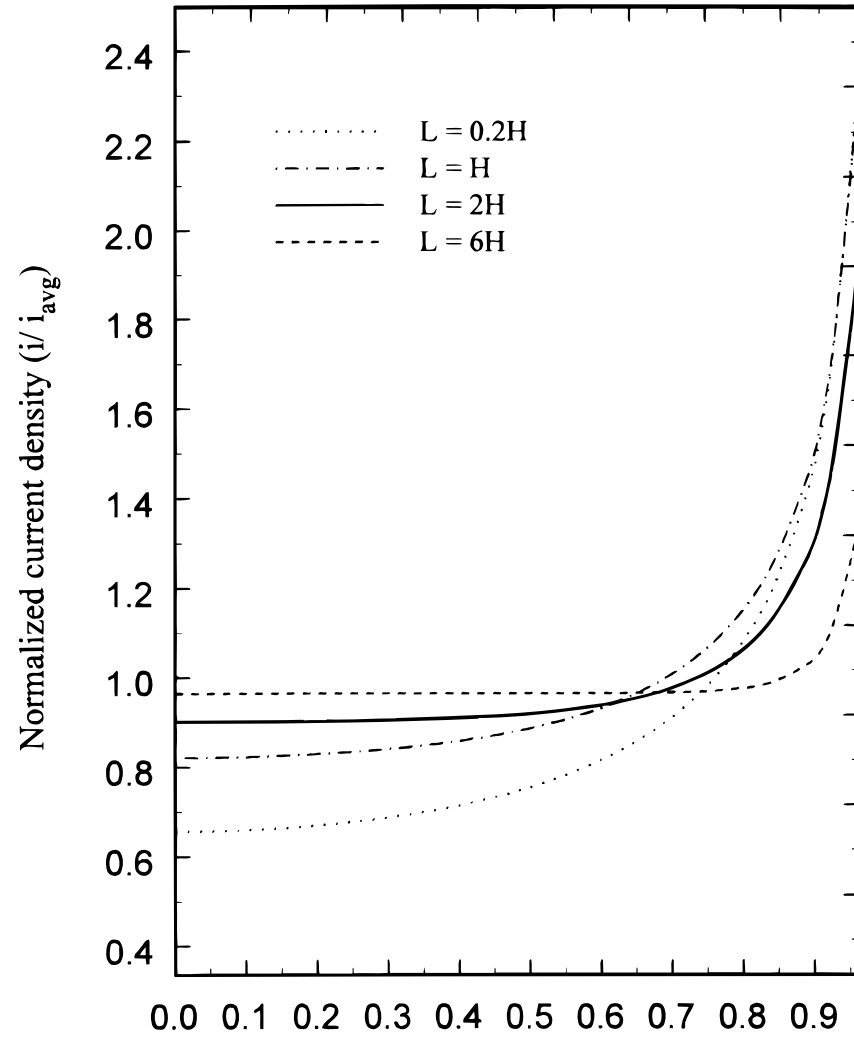

Dimensionless distance from the center $(2 x / L)$

Figure 10. Primary current distribution on plane parallel electrodes, effect of aspect ratio $(2 H / L)$.

The same transformation used above (Eq. 61) is used on the $r$ coordinate in this example. The transformation changes the derivatives in $r$ only. Hence, the resulting equation is solved by integrating analytically in $y$ after discretizing in transformed $r$, and the primary current density distribution on the disk electrode is plotted in Fig. 11 using $N=25$ node points ( 25 on the disk and only 5 on the insulator). For conserving time, $z$ is integrated to a numerical value (say 10) and increased only if necessary instead of finding the limit $z$ tending to infinity. Time taken for generating the profile is around $100 \mathrm{~s}$. It should be noted that Laplace's equation is discretized in $r$ and not in $z$. If Laplace's equation is discretized in $z$, then the coefficient matrix $A$ becomes $r$ dependent and the solution is not given by an exponential matrix but by the matrizant. ${ }^{10}$

\section{Other Examples}

The technique presented here can also be used to predict primary and secondary current density distributions on a sinusoidal profile. ${ }^{15}$ The potential is governed by the Laplace equation

$$
\frac{\partial^{2} \Phi}{\partial Y^{2}}+A^{2} \frac{\partial^{2} \Phi}{\partial X^{2}}=0
$$

where $A$ is the ratio of amplitude to the length of the wave subject to the boundary conditions

$$
\begin{aligned}
& \Phi=0 \text { at } Y=-\cos (2 \pi X) \text { (at the sinusoidal surface) } \\
& \frac{\partial \Phi}{\partial Y}=-1 \text { as } Y \rightarrow \infty \text { (counter electrode) } \\
& \frac{\partial \Phi}{\partial Y}=0 \text { as } X=0 \text { and } \frac{1}{2} \text { (symmetry condition) }
\end{aligned}
$$

This BVP (Eq. 63 and 64) is discretized in $X$ and integrated analytically in $Y$. It should be noted that for this BVP, the initial condition 


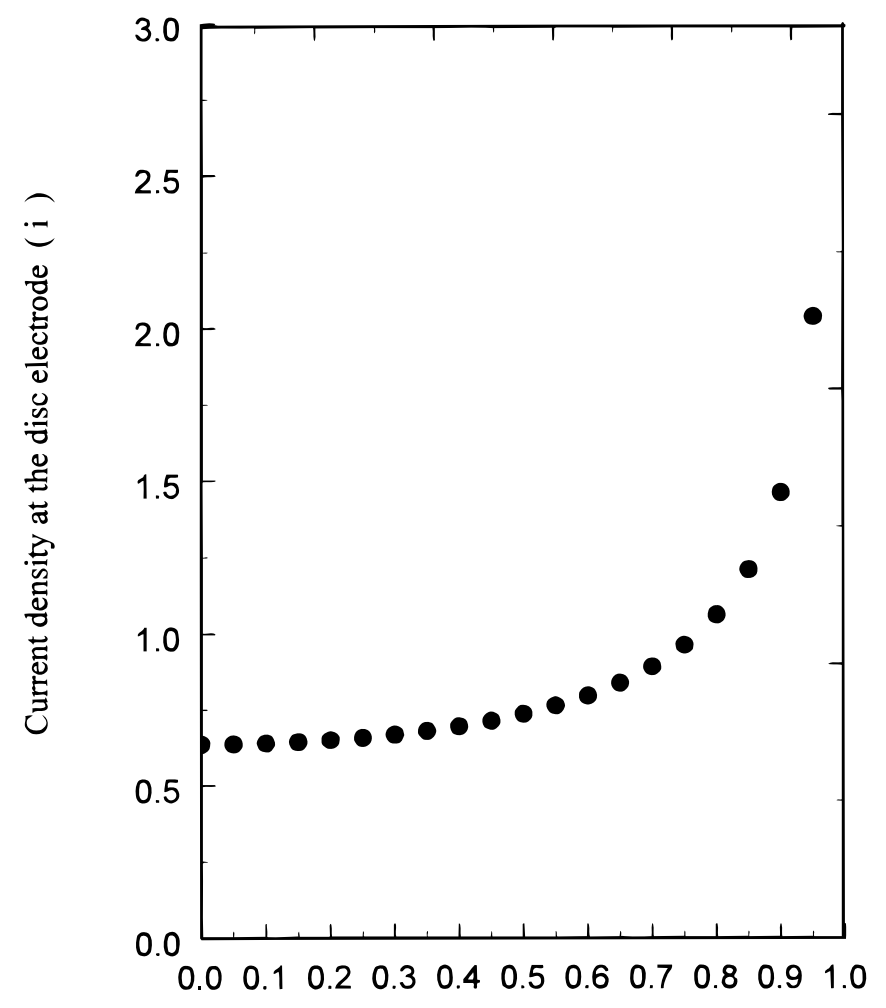

Dimensionless distance from the center, $r$

Figure 11. Primary current distribution on a RDE, semianalytical method for semi-infinite domains.

vector $\mathbf{Y}_{0}$ is completely unknown. Therefore, both the potential and its derivative are taken to be constants at $Y=0$. That is, we solve for both $p_{\mathrm{i}}$ and $c_{\mathrm{i}}$ at the node points at $Y=0$. There are $2 N$ constants to be determined. $N$ equations are given by the boundary condition at $Y \rightarrow \infty$. The other $N$ equations are given by substituting the numerical value for the position of the sinusoidal surface (as in a Hull cell) and using the boundary condition at the sinusoidal electrode. Results obtained for $A=0.1, N=20$ node points are in excellent agreement with the reference. The $Y$ component of the current density, relative to the applied current at the counter electrode, is plotted in Fig. 12. The total time taken by Maple for this BVP is around 1 min.

We used our method to solve several other examples

1. Plane parallel electrodes separated by an infinite distance. ${ }^{16}$

2. Infinitely long plane parallel electrodes separated by a narrow gap. $^{16}$

3 . Potential profiles in a two-dimensional rectangular electrolyzer (section 6.3 .3 of Ref. 14).

4. Cylindrically symmetric potential-distribution problem. ${ }^{17}$

5. Potential distribution in a flow-by electrode for the mass-transfer-controlled current. ${ }^{18,19}$

6. Interpretation of measured polarization resistance at a solid electrode/electrolyte interface. ${ }^{20}$

7. Estimation of front velocity in electrodeposition onto highly resistive substrates. ${ }^{21}$

The details associated with these examples are available from the authors upon request.

\section{Limitations}

A semianalytical technique is presented to solve Laplace's equation. To integrate analytically using the exponential matrix, Laplace's equation should have constant coefficients in $y$. For example, Laplace's equation in cylindrical coordinates can be integrated in $z$ but not in $r$. To integrate in $r$, the matrizant ${ }^{10}$ should be used. If both the independent variables appear explicitly in Laplace's equation as in elliptic coordinates, the matrizant method must be used. It is not ad-

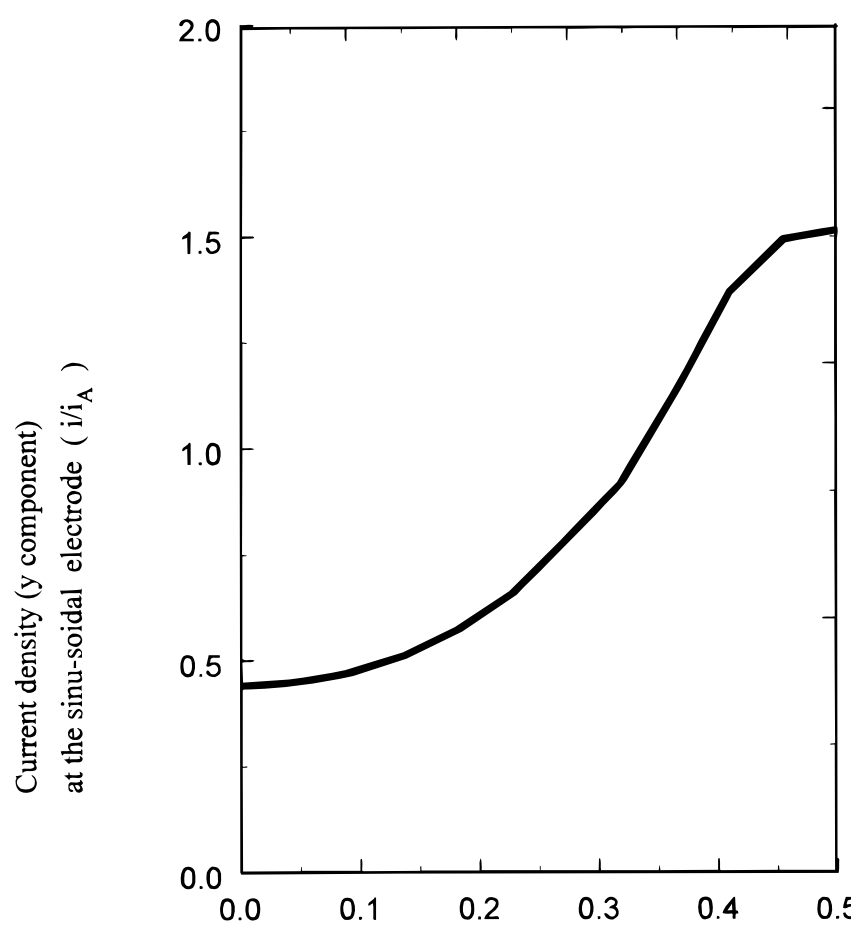

Dimensionless distance from the valley, $\mathrm{X}$

Figure 12. Primary current distribution on a sinusoidal profile, semianalytical method for curved electrodes.

visable to go beyond $N=20$ node points. Also, Maple V is a memory intensive software compared to a numerical language like FORTRAN. One should have a PC with a minimum RAM of $64 \mathrm{MB}$ for the problems discussed here. Also, only Laplace's equation is solved in this paper. Tertiary current distributions would require finding the exponential matrix only once, but the math involved in the manipulation is complicated and will be communicated in a later paper.

\section{Discussion}

In this paper, we have presented a general solution technique for solving current and potential distribution problems. The method presented here can be used easily to determine both primary and secondary current density distributions (both linear and nonlinear by solving just once). The method developed is more general than analytic techniques (valid for nonlinear boundary conditions also) and gives better insight than numerical techniques (solve just once for any boundary condition on the cathode and anode) for a certain class of problems. Laplace's equation is a partial differential equation; we converted it to a linear set of ordinary differential equations (ODEs), the solution for which is general and does not depend upon the boundary conditions and shapes in $y$. The method presented here is also useful from a pedagogic point of view because it is more general than analytic methods (e.g., using the solution of a rectangle to predict current distributions in a Hull cell). Also, this technique could be extended to predict tertiary current density distributions. Also, the technique presented here could be extended to treat nonlinear problems in a manner similar to that presented earlier. ${ }^{10} \mathrm{An}$ alternative to finding the exponential matrix is developed by Subramanian and White 22 and will be used for solving current and potential distributions symbolically and communicated later.

It is hoped that this paper will serve as a useful tool for anyone who wants to solve current density distribution problems. For improving the speed of computations, finite differences of higher order of accuracy are used for discretization along the $x$ axis. We have developed a general Maple program in which the user can specify the order of accuracy. The user has to specify only the governing equations and boundary conditions. Once these are specified, the program can be 
executed to obtain the potential and current density profiles. Maple programs used for obtaining the specific results in this paper and the other examples mentioned are available on a diskette and can be obtained upon request from the authors.

\section{Acknowledgments}

The authors are grateful for the financial support of the project by the Office of Research and Development (ORD) under contract no. 93-F 148100 - 100. Also, one of the authors (V.R.S.) would like to thank Dr. N. G. Renganathan (Scientist, CECRI, Karaikudi, India) for suggesting the examples.

The University of South Carolina assisted in meeting the publication costs of this article.

\section{List of Symbols}

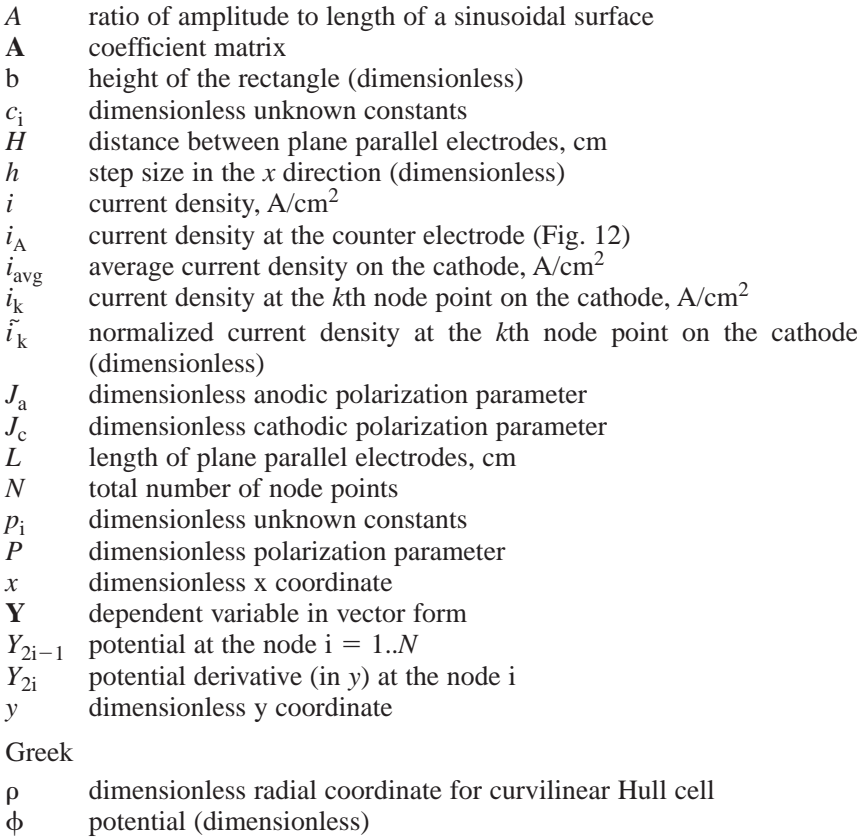

$\psi \quad$ dimensionless potential inside a curvilinear Hull cell

$\nabla \quad$ gradient (dimensionless)

$\theta \quad$ angular position in a curvilinear Hull cell

Subscripts and superscripts

$0 \quad$ initial condition, at $y=0$

$\mathrm{i} \quad$ index of the node point

$\mathrm{k} \quad$ index of the node point on the cathode

\section{References}

1. J. S. Newman, Electrochemical Systems, Chap. 18, Prentice Hall, Englewood Cliffs, NJ (1991).

2. A. C. West and J. S. Newman, in Modern Aspects of Electrochemistry, Vol. 23 , B. E. Conway, J. O'M. Bockris, and R. E. White, Editors, p. 113, Plenum Press, New York (1992).

3. G. Prentice, Electrochemical Engineering Principles, p. 195, Prentice Hall, Englewood Cliffs, NJ (1986).

4. J. S. Newman, in Electroanalytical Chemistry, Vol. 6, A. J. Bard, Editor, p. 187, Marcel Dekker, Inc., New York (1973).

5. R. Varma and J. R. Selman, Characterization of Electrodes and Electrochemical Processes, Chap. 13, John Wiley \& Sons, Inc. New York (1991).

6. P. De Vidts and R. E. White, Comput. Chem. Eng., 16, 1007 (1987).

7. M. E. Davis, Numerical Methods and Modeling for Chemical Engineers, p. 128 , John Wiley \& Sons, New York (1984).

8. C. L. Smith, R. W. Pike, and P. W. Murill, Formulation and Optimization of Mathematical Models, p. 381, International Textbook Company, Scranton, PA (1970).

9. A. Varma and M. Morbidelli, Mathematical Methods in Chemical Engineering, p. 56, Oxford University Press, New York (1997).

10. V. R. Subramanian, B. S. Haran, and R. E. White, Comput. Chem. Eng., 23(3), 287 (1999).

11. V. R. Subramanian and R. E. White, in Tutorials in Electrochemical EngineeringMathematical Modeling, R. F. Savinell, J. M. Fenton, A. C. West, S. L. Scanlon, and J. Weidner, Editors, PV 99-14, p. 36, The Electrochemical Society Proceedings Series, Pennington, NJ (1999).

12. J. Lee and T. W. Chapman, J. Electrochem. Soc., 145, 3042 (1998).

13. R. Morris and W. Smyrl, J. Electrochem. Soc., 136, 3229 (1989).

14. I. Rousar, K. Micka, and A. Kimla, Electrochemical Engineering 1, Elsevier, New York (1986).

15. P. S. Fedkiw, J. Electrochem. Soc., 127, 1305 (1980).

16. C. Wagner, J. Electrochem. Soc., 98, 116 (1951).

17. J. B. Riggs, An Introduction to Numerical Methods for Chemical Engineers, p. 277, Texas Tech University Press, TX (1988).

18. P. S. Fedkiw, J. Electrochem. Soc., 128, 831 (1981).

19. P. S. Fedkiw, Comput. Chem. Eng., 6(4), 327 (1982).

20. A. M. Svensson and K. Nisancioglu, J. Electrochem. Soc., 146, 1840 (1999).

21. P. Desprez, M. Matlosz, J. D. L. Yang, and A. C. West, J. Electrochem. Soc., 145, 165 (1998).

22. V. R. Subramanian and R. E. White, Comput. Chem. Eng., Submitted. 